\title{
Intracavity Gain Detection Applied to Transient Inversions of IF
}

\author{
R. L. WILLIAMSON*, L. HANKO AND S. J. DAVIS‡
}

Air Force Weapons Lab., Kirtland AFB Albuquerque, New Mexico 87117, USA

A CW dye laser has been used to study transient optical gain on the $\mathrm{B} \rightarrow \mathrm{X}$ electronic transition of iodine monofluoride. By placing the gain generator inside the $\mathrm{CW}$ dye laser cavity, gain was readily observed even at levels too small to support lasing in a low loss cavity. Transient phenomena, including V-T energy transfer, were easily observable. An analysis of the intracavity gain detection technique revealed a method for estimating optical gain. The technique should be readily applicable to other systems.

KEY WORDS: Intracavity, transient inversions, IF.

\section{INTRODUCTION}

The search for a visible chemical laser system has been an ongoing research task for more than a decade. During this time numerous papers describing chemiluminescence and kinetic studies with the stated goal of laser development have appeared in the literature ${ }^{1,2}$. In addition, there have been reports of optically and electrically pumped lasers on molecules that are themselves attractive candidates for chemical lasing ${ }^{3-6}$.

Despite the considerable activity in the area, there has yet to be a successful visible chemical laser demonstration. This stems from the inherently more difficult problem of efficiently channeling chemical energy into electronically excited states, in contrast to the analogous

* Current address: Sandia National Laboratories, P.O. Box 5800, Albuquerque, NM 87185.

$\ddagger$ Current address: Physical Sciences Inc., P.O. Box 3100, Research Park, Andover, MA 01810. 
process of chemically pumping vibrational states of molecules (e.g. $\mathrm{HF}, \mathrm{CO}$ ) which produce efficient infrared laser systems.

As one attempts to develop such systems, sensitive diagnostic tools are invaluable. Obviously, a critical parameter in any potential laser system is the magnitude of the small signal gain (SSG). Since no visible chemical lasers have yet been demonstrated, we can safely conclude that the SSG in these systems is very small. A versatile, yet simple, SSG diagnostic would be a valuable tool in characterizing the laser potential of candidate systems.

The extreme sensitivity of the intracavity gain detection technique has recently been demonstrated by Truesdell and $\mathrm{Keller}^{7,8}$. They were able to show that the technique was capable of detecting net optical gains estimated to be as small as $\sim 2 \times 10^{-5}$ in a $\mathrm{CW}$ system. Though other highly sensitive gain diagnostics are available ${ }^{9-12}$ they invariably require that the probe laser wavelength exactly match the wavelength of the inverted transition under investigation. This requirement is eliminated when using intracavity gain detection due to the observation that whenever the probe laser is tuned near a gain transition, part, or all, of the the probe output "pulls" to and "locks" onto the gain transition wavelength, depending on how well the probe laser wavelength matches the gain wavelength and the degree of inhomogeneity in the probe laser gain medium. This wavelength "pulling" phenomenon ${ }^{7,8,13,14}$ makes the intracavity technique an

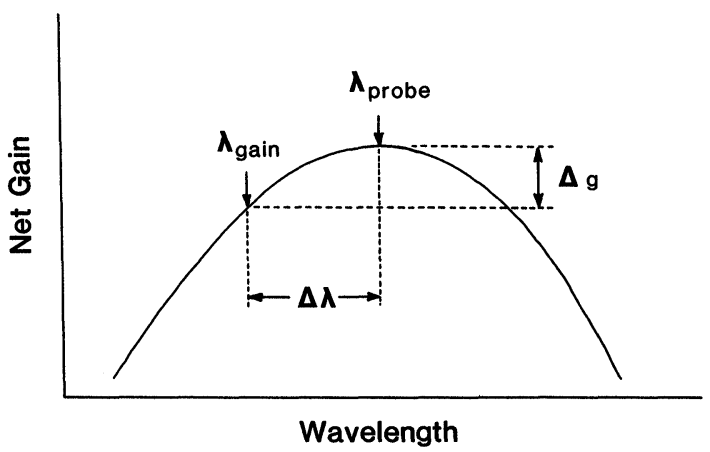

Figure 1 A depiction of the tuning element transmittance curve convoluted with the dye gain curve. The dye laser oscillates at the maximum. If test gain is supplied at a wavelength $\Delta \lambda$ away, the output of the dye laser will "pull" or "lock" onto the test gain wavelength provided the test gain is $\geqq \Delta g$. 
extremely versatile tool for gain detection and optimization studies in any medium which exhibits potential or known laser transitions within the wavelength range of the probe laser.

In this paper we describe the application of this technique to the study of a pulsed gain system. The technique is used to study gain conditions in optically pumped, transient inversions of the IF $B \rightarrow X$ system. (The characteristics of this system which make it an attractive chemical laser candidate have been described in detail elsewhere ${ }^{15,16}$.) The method proved to be especially useful as it provided detailed temporal data on relative gain magnitude as a function of pressures and flow rates of both reactant gases and carrier gases in the flow reactor cell.

\section{SOME COMMENTS ON THE INTRACAVITY GAIN DETECTION TECHNIQUE}

The intracavity gain detection technique involves placing the test medium directly within the cavity of an operating dye laser and using the dye gain to offset all steady-state losses in the cavity. As noted above, the two principal advantages of using intracavity gain detection are enhanced sensitivity and the lack of a requirement to exactly match the wavelength of the inverted transition. The sensitivity enhancement is well understood and adequate discussions are available ${ }^{17,18}$. However, an understanding of the wavelength "pulling" phenomenon is important to understanding many of the dynamic processes present when applying the technique to pulsed systems, and will be briefly discussed in this section.

As mentioned in the Introduction, when gain is created in a test cell placed within the probe laser cavity, a fraction of the output of the dye laser may be "pulled" to the gain transition wavelength. This happens provided the test gain is sufficiently large to cause the wavelength at which it occurs to be the wavelength of least loss and, thus, of highest Q. The principle involved is illustrated in Figure 1. The curve shown in this figure represents the convolution of the dye gain curve and the transmittance curve of the dye laser tuning element. As is apparent from the figure, the magnitude of the pulling effect, that is the wavelength range $\Delta \lambda_{\max }$ over which the dye laser output will "lock" onto the gain wavelength, is a function of the magnitude of the gain created in the test medium. Thus, it is generally observed that as 
experimental parameters are changed to increase net gain (e.g. through increasing the pump power or optimizing reactant gas flows), the probe laser may be "pulled" onto the gain transition wavelength over a broader wavelength region.

In experimental situations where one is interested in detecting SSG, it is convenient to set up conditions so that the probe laser is as sensitive as possible to wavelength "pulling." This implies that the curve in Figure 1 should be made as broad as possible. The tuning element used in the experiments described in this paper was a single birefringent plate made of crystalline quartz, cut such that the optic axis was parallel to the crystal surface. The plate was placed in the cavity of the laser at Brewster's angle with respect to the beam. The dye laser output was tuned by rotating the plate about an axis normal to its surface. Such a rotation varies the angle, $\phi$, between the wave-normal vector of the beam and the optic axis, thus changing the wavelength of light which will pass through the Brewster surfaces unattenuated ${ }^{19}$. Figure 2 shows the transmittance curve, $\Lambda(\phi)$, of the plate measured at a tuning angle of $34^{\circ}$. Note that the transmittance curve is very broad due to the fact that a single plate was used rather than a multi-plate tuning element. This has the desired effect of making the probe laser more sensitive to "pulling." Of course, if the tuning element curve is made too broad, it will not tune the laser output effectively and this imposes an inherent limitation on the maximum sensitivity of the probe laser to the "pulling" phenomenon.

From what has been said to this point, it seems that it should be possible to make gain estimates based on the magnitude of $\Delta \lambda_{\max }$. For example, assuming that the gain curve over this wavelength region $\left(\Delta \lambda_{\max }\right)$ is relatively flat, it is easy to see that the transmittance curves of the quartz plate may be used to estimate the net gain created in the test medium. As an example, using the empirically determined curve in Figure 2, if it were determined that under certain experimental conditions the gain of the test medium could "pull" the dye laser a maximum of $\sim 50 \AA$ from a probe wavelength of about $5900 \AA$ and lock it onto the wavelength of a gain transition bandhead at $5950 \AA$, we would conclude that the test medium had to supply a net gain at least equal to or greater than the wavelength dependent loss introduced by the plate at this point, namely $\sim 1 \%$. However, there are various complicating factors which make this method of measurement useful only for making rough estimates of SSG. 


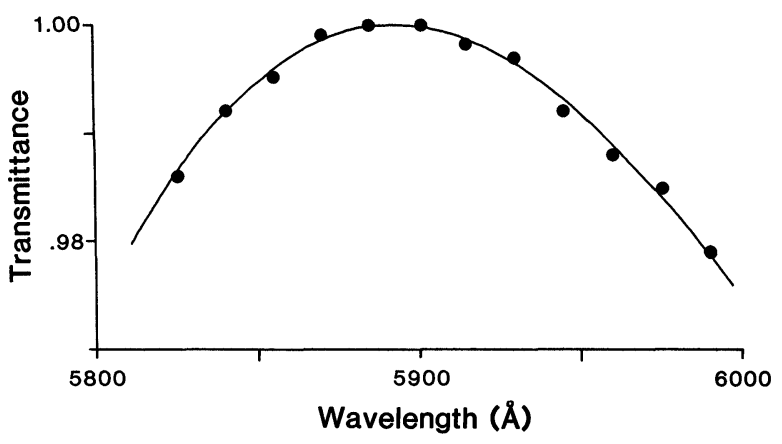

Figure 2 The transmittance curve of a single quartz plate tuning element inserted into the beam at Brewster's angle at a tuning angle of $34^{\circ}$. The tuning angle, $\phi$, is defined as the angle between the optic axis and the intersection of the plane of incidence with the cyrstal surface. As $\phi$ is adjusted the transmittance curve maximum shifts in wavelength changing the point of maximum $Q$ of the cavity and tuning the laser output.

First, if the experiment is set up to maximize the sensitivity of the probe laser to wavelength "pulling," this implies that the tuning element transmittance curve and the dye gain curve have similar widths. Thus, the assumption concerning the relative flatness of the gain curve is not valid. This is most easily seen by noting that the laser does not oscillate at the maximum of $\Lambda$, but at the maximum of the convolution curve. Calculations suggest, and observations confirm, that for the conditions of interest (single plate tuning element, rhodamine 590 dye, $\phi=25^{\circ}-45^{\circ}$ ) the difference between the two curve maxima may be quite large, $\sim 200 \AA$. If the gain curve were "flat" relative to the tuning element transmittance curve, as would be the case if a high quality, multiplate tuning element were used, the laser would oscillate at the transmittance curve maximum. The task of having to generate the full convolution curve for each tuning angle of interest complicates the gain measurement process considerably. However, even if this were done, there are other complications which make the method highly system dependent.

It is well-known that the probe laser is very sensitive to intracavity absorptions ${ }^{17,18,21,22}$. In any realistic chemical laser system there will be a number of gas phase species which may absorb in the spectral region of interest. For example, in the generation of IF, unreacted $I_{2}$ is present in the cell (see discussion below). Figure 3 shows the effect on the probe laser output of adding 300 mTorr of unreacted molecular 


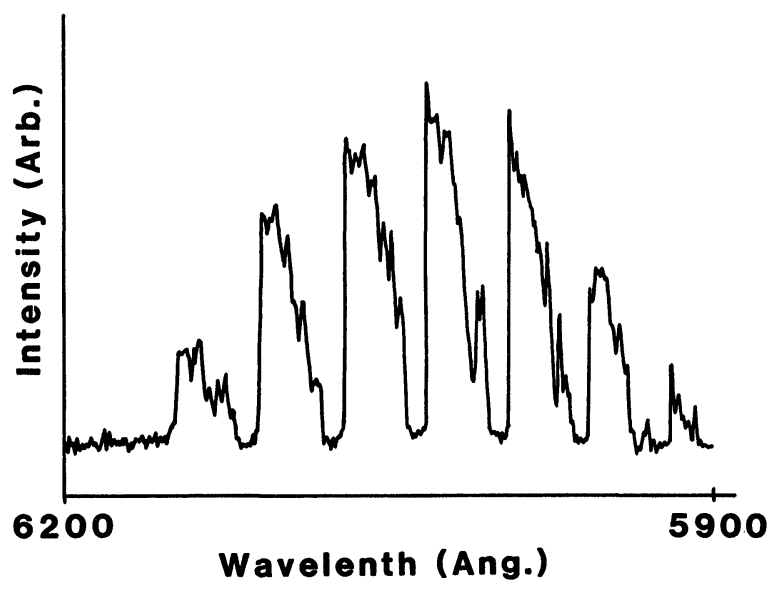

Figure 3 The probe laser output intensity as a function of wavelength, illustrating the effects of intracavity absorptions. This intracavity absorption spectrum was obtained by filling the test cell with approximately $300 \mathrm{mTorr}$ of $I_{2}$ and then monitoring the dye laser output intensity while scanning the laser over its entire wavelength range. The dye used for this experiment was Rhodamine 590.

iodine to the test cell. If the probe laser is set to an absorption peak of $\mathrm{I}_{2}$ before $I_{2}$ is admitted into the test cell, when the $I_{2}$ is bled in the probe will shift to a new wavelength where the absorption loss is smaller. Obviously, the presence of intracavity absorbants may play a major role in determining the nature of the gain curve, and thus the magnitude and nature of the wavelength "pulling" effect. Thus, in general it is difficult to extract accurate gain measurement data from intracavity gain detection experiments, except perhaps in instances where simple, well characterized systems are being studied.

Finally, it is easily envisioned that the presence of absorbing species in the test cell may give rise to various interfering transient effects in pulsed gain experiments in the event that some of these species also absorb in the wavelength region of the pump pulse. This situation will be discussed in the Results section of this paper.

\section{EXPERIMENTAL}

A schematic of the experimental is shown in Figure 4. The cavity of the Coherent Model CR590 CW dye laser was extended about one meter 
to incorporate a $64 \mathrm{~cm}$ longitudinal flow cell. The flow reactor cell is necessary when working with IF since this radical is chemically unstable. The birefringent quartz plate used as the probe laser tuning element in these experiments was obtained by modifying a stock three-element filter supplied by Coherent. The dye used for these experiments was rhodamine 590 in ehtylene glycol. A Phase-R Model DL-1400 flashlamp pumped dye laser was used to produce population inversions in the test medium. This laser has a pulse width of about $200 \mathrm{~ns}$ and is capable of output energies as high as $150 \mathrm{~mJ} / \mathrm{pulse}$ when using coumarin 480 dye (Exciton Chemical Co.). Typically, however, pulse energies between 20 and $50 \mathrm{~mJ}$ were used. The pulse rate was typically $0.3 \mathrm{~Hz}$.

To prevent the dye laser pulse from hitting the dye jet of the CW probe laser and interfering with its operation, it was necessary to insert a glass long pass filter (Oriel Model No. 51500) into the dye laser cavity as shown in Figure 4. The filter was inserted at Brewster's angle and

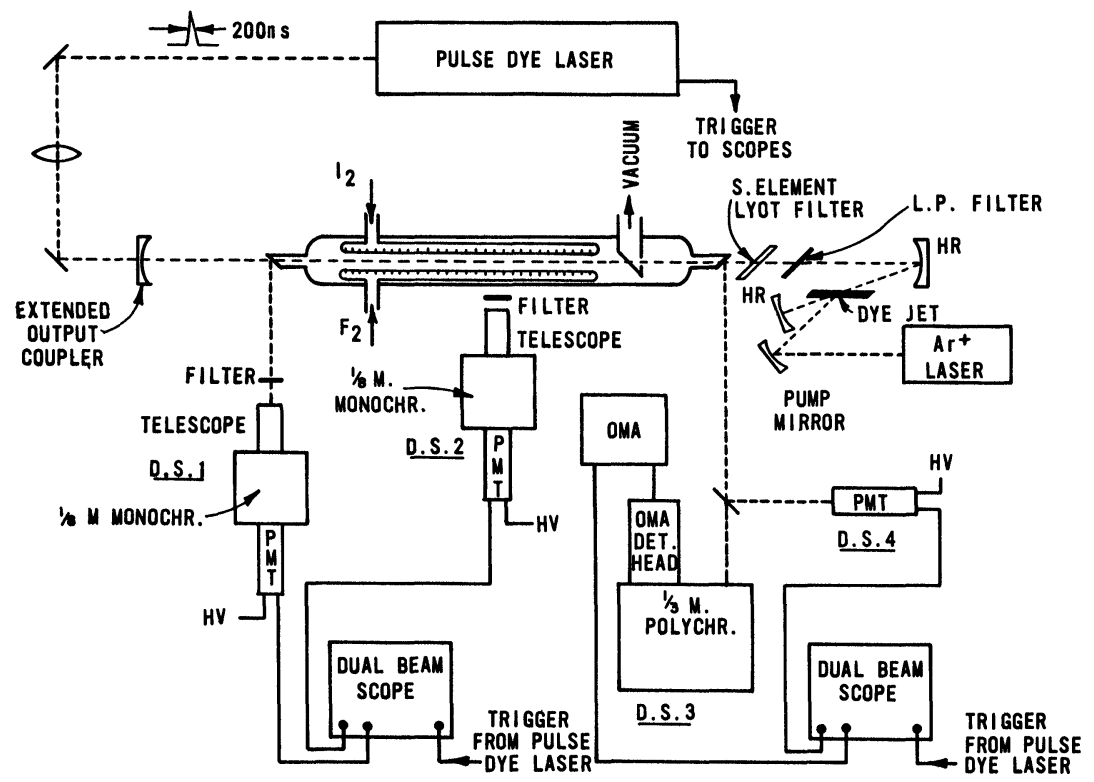

Figure 4 The pulsed intracavity gain diagnostic apparatus. DS-1 (Detection System 1) was used to detect spectrally resolved gain, DS-2 to monitor the IF side fluorescence, DS-3 to monitor the probe laser wavelength, and DS-4 to detect net or spectrally unresolved gain pulse. 
had a cut-on wavelength of $5300 \AA$. Even at modest pump powers this effect can be quite large. The focused beam of the pump laser easily vaporizes the probe laser dye jet, disrupting probe laser operation on the millisecond time scale. Attempting to circumvent the problem by shifting the pump beam off-axis seriously degrades the gain pulse signal, while lowering the pump power not only lowers the signal intensity but also gives rise to spurious gain signals since the dye often absorbs at the pump wavelength. To date, the insertion of the long pass filter into the cavity has proved to be the most convenient solution to this problem. Local heating of the filter by the pump pulse can cause transients in the $\mathrm{CW}$ output of the probe laser and pump laser powers were held low to minimize these effects.

As illustrated in Figure 4, transients in the probe laser intensity were monitored by detecting the low power reflections from the Brewster window surfaces at each end of the flow reactor cell. The detection system was set up so that both temporal and spectral changes in the probe laser could be resolved. Detection system 1 (DS-1) was used to monitor spectrally resolved gain pulses. The detection system employed an Oriel Model 7240 1/8-meter grating monochromator with a 1200 groove $/ \mathrm{mm}$ grating blazed at $500 \mathrm{~nm}$ with a reciprocal dispersion of $64 \AA / \mathrm{mm}$. Ultimate resolution of this system was $5 \AA$. The monochromator was coupled to a Oriel Model 77340 photomultiplier. The output of this system was monitored simultaneously with that of DS-2 on a Tektronix Model 7844 dual beam oscilloscope. DS-2 was used to detect the time resolved side fluorescence and employed similar optical components. Net (spectrally unresolved) gain pulses were detected using an appropriate long pass filter (to eliminate interference from the pump pulse) and an RCA Model 4832 PMT (DS-4). The probe laser was tuned to the desired wavelength region with the aid of DS-3 which consisted of an optical multichannel analyzer (SSR Model 1250A with a 1205 DE detector head) coupled to a McPherson Model 218 1/3-meter monochromator. The monochromator was equipped with a 1200 groove $/ \mathrm{mm}$ grating blazed at $6000 \AA$ and has an ultimate resolution of better than $1 \AA$. The outputs of detection systems 3 and 4 were fed to a Tektronix Model 7704 oscilloscope.

Normally, Tektronix Model 7A22 plug-in amplifiers $(1 \mathrm{MHz}$ bandpass, $<10 \mu \mathrm{V}$ sensitivity) were used in the oscilloscopes, with the inputs shunted to ground through $500 \mathrm{ohm}$ resistors. This 
configuration was sensitive and gave smooth, practically noise free signals, but the $350 \mathrm{~ns}$ risetime gave rise to modestly distorted pulse shapes. Under conditions where sensitivity was not an issue and an accurate record of the temporal evolution of the gain pulse was required, Tektronix Model 7A19 plug-in amplifiers were used in the oscilloscopes. With these plug-ins it was necessary to preamplify the gain signal with a Hewlitt Packard Model 461A amplifier. The bandpass of this instrumentation was limited to the bandpass of the preamp at $150 \mathrm{MHz}$. This "fast" detection system was considerably less sensitive and gave rise to considerably more noisy signals.

The gain detection apparatus was used to detect optically pumped gain on various $v^{\prime} \rightarrow v^{\prime \prime}$ transitions of the IF (B $\rightarrow \mathrm{X}$ ) system. (The IF $\mathrm{B} \rightarrow \mathrm{X}$ laser system has been described elsewhere ${ }^{4,16}$.) With the flow reactor empty the probe laser was brought to threshold and used to align the pump beam to the axis of the $\mathrm{CW}$ dye laser. It is critical that the probe beam be contained within the volume of the pump beam. Iodine and fluorine were then admitted into the intracavity test cell and the flows were adjusted to maximize IF production at the desired total pressure. Helium was used as the carrier gas for the iodine. Detection system 1 was then tuned to the bandhead of the $B \rightarrow X$ emission band that was to be probed for gain, and the test medium was optically excited with the pump laser. Vibrational levels $v^{\prime}=2,3$ and 4 were pumped in these experiments. The pump laser was then fine tuned to maximize the bright yellow $\mathrm{B} \rightarrow \mathrm{X}$ side fluorescence detected with DS-2. At this point gain pulses were usually obtained on both detection systems 1 and 4 . DS- 1 was usually scanned in the region of the emission bandhead to find the wavelength where the gain was at its maximum. The spectrally unresolved gain monitored with DS -4 could be maximized by tuning the probe laser.

\section{RESULTS}

An example of the type of data collected in the pulsed gain detection experiment is shown in Figure 5. The gain was detected with DS-1 ("fast" detection electronics) at $6252 \AA$ (10 $\AA$ bandpass) corresponding to the $0^{\prime}-5^{\prime \prime}$ transition, with the probe laser having been set at $6205 \AA$. Thus, in this example the probe laser has been "pulled" nearly $50 \AA$ onto the gain transition wavelength. The pump energy for this experiment was held at $\sim 50 \mathrm{~mJ}$. Under optimum conditions, and 
in the pressure range considered here, the IF gain could pull the probe laser output a maximum of 50 to $55 \AA$. From the curve shown shown in Figure 2, this indicates an estimated net gain of $\sim 1$ percent, or a gain coefficient of $\sim 3 \times 10^{-4}$ (40 cm "active" cell length). Table I shows various example conditions under which gain was detected on collisionally pumped transitions in our system. We found that "strong" gain pulses could be obtained at total cell pressures ranging from 20 to $100 \mathrm{mTorr}$ provided the ratio of $F_{2}$ to $I_{2}$ was held in the range of 45 to 50.

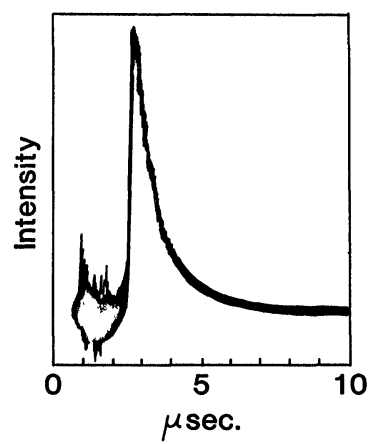

Figure 5 "Thermalized" IF $\mathrm{B}\left(v^{\prime}=0\right)-\mathrm{X}\left(v^{\prime \prime}=5\right)$ gain pulse obtained by pumping the $3^{\prime}-0^{\prime \prime} B \leftarrow X$ transition at $\sim 4965 \AA$. The probe laser was set at $6205 \AA$ and the gain pulse was monitored with DS- 1 at $6252 \mathrm{~A}$, the bandhead for the $0^{\prime}-5^{\prime \prime}$ transition. The total cell pressure was 22 Torr. This spectrum was obtained using the "fast" electronics described in the text to resolve the true temporal lineshape. The pump pulse occurs approximately $600 \mathrm{nsec}$ subsequent to the trigger.

Table I Conditions for which gain was optimized on the $0^{\prime}-4^{\prime \prime}$ transition of IF subsequent to pumping the $3^{\prime}-0^{\prime \prime}$ transition.

\begin{tabular}{lll}
\hline $\mathrm{P}_{\text {tot }}$ (Torr) & $\mathrm{F}_{2} / \mathrm{I}_{2}$ & Flow Vel. $(\mathrm{cm} / \mathrm{s})$ \\
\hline 99.8 & $\sim 45$ & 45 \\
72.9 & $\sim 48$ & 61 \\
48.5 & $\sim 45$ & 93 \\
23.6 & $\sim 48$ & 194 \\
15.0 & $\sim 43$ & 310 \\
8.5 & $\sim 37$ & 570 \\
24.7 & $\sim 11^{\mathrm{a}}$ & 180 \\
25.2 & $\sim 160^{\mathrm{b}}$ & 160 \\
\hline
\end{tabular}

\footnotetext{
${ }^{a}$ Lowest value of the ratio of $F_{2}$ to $I_{2}$ for which gain was detected at a total cell pressure of $\sim 25$ Torr.

${ }^{b}$ Highest value of the ratio of $F_{2}$ to $I_{2}$ for which gain was detected at a total cell pressure of $\sim 25$ Torr.
} 
It is interesting to note that IF is the only interhalogen for which lasing has been demonstrated from "thermalized" or collisionally relaxed $v^{\prime}, J^{\prime}$ states $^{16}$. Because the inversion is achieved through collisional relaxation, we expect that the onset of the gain should change as a function of the cell pressure. Figure 6 shows that the onset of gain decreases as the total cell pressure, and thus the collision rate, is increased. This data compares favorably with that obtained in a previous study of the pressure dependence of the delay of the onset of lasing in a collisionally pumped IF laser ${ }^{16}$.

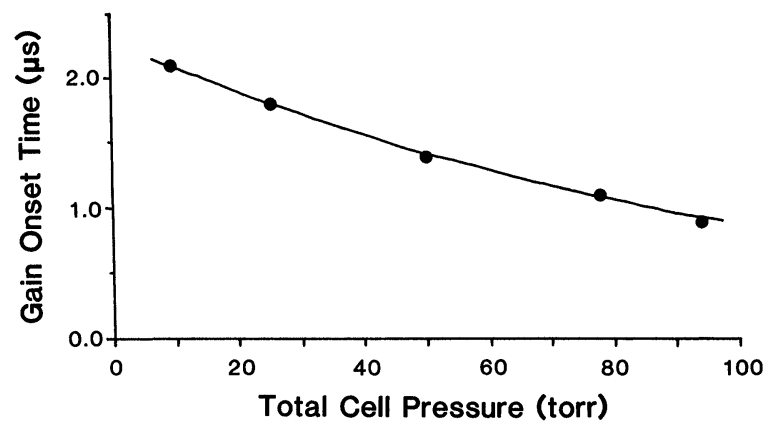

Figure 6 The time required for "thermalized" gain onset as a function of total cell pressure. This data was collected with the probe laser set approximately $20 \AA$ to the blue of the $0^{\prime}-5^{\prime \prime}$ bandhead, at $6231 \AA$.

"Thermalized" gain could only be detected on B $\rightarrow \mathrm{X}$ transitions involving the $v^{\prime}=0$ level. Gain on transitions such as the $2^{\prime}-5^{\prime \prime}$ $\left(5953 \AA \text {, Franck-Condon factor }\left(q_{2-5}\right)=0.096\right)^{20}$ or the $1^{\prime}-6^{\prime \prime}(6318 \AA$, $\left.q_{1-6}=0.063\right)^{20}$ could not be detected despite favorable Franck-Condon factors and computer predictions of reasonable transient populations at relatively low total pressures. This observation corroborates the results of earlier attempts to obtain lasing from these intermediate collisionally pumped levels ${ }^{16}$. This is evidence of very efficient $\mathrm{V}-\mathrm{T}$ transfer in this system, causing the residence time in any intermediate $v^{\prime}$ level to be short relative to the stimulated emission lifetime.

Under normal conditions scanning DS-1 revealed that the "thermalized" gain pulse did not occur only at the bandhead but was spread over a broad region ( $\sim 25 \AA$ at $50 \mathrm{~mJ} /$ pulse pump energy) of the band. This range became increasingly narrow as pump energies were 
lowered and could also be narrowed by tuning the probe laser further from the emission bandhead. Near threshold gain could only be detected in the immediate vicinity of the emission bandhead. When the probe laser was scanned onto the emission band with the spectrometer bandpass set such that the probe laser wavelength was not detected, gain pulses were detected at various points throughout the band illustrating that inversions were being created simultaneously in many rotational levels. Although we were not able to resolve gain from individual rovibronic lines, it was apparent from these experiments that the gain was spread over many rovibronic transitions.

When the total cell pressure was lowered to less than about 7 Torr, the thermalized gain disappeared. Gain on optically pumped (as opposed to collisionally pumped) $v^{\prime}, J^{\prime}$ levels appeared at cell pressures below about 5 Torr. This "direct" gain was investigated primarily on the $3^{\prime}-7$ " transition at $6229 \AA$. The "direct" gain was much weaker than the "thermalized" gain for the cell configuration used and could only be observed after considerable effort and under optimum conditions. Under the most favorable circumstances the "direct" gain could pull the probe laser output of the order of $25 \AA$ (onto the bandhead from the blue), indicating a net estimated gain of $\sim 0.3$ percent, or a gain coefficient of about $8 \times 10^{-5} / \mathrm{cm}$. Under conditions more representative of the norm, the probe output could only be pulled 5-10 $\AA$, indicating a net gain of $<0.1$ percent. Efforts to lase IF on a "direct" transition under these conditions failed, even at significantly higher pump powers, illustrating that the technique is sufficiently sensitive to detect subthreshold gain. Attempts to detect both "direct" and "thermalized" gain simultaneously at the same cell pressure were not successful.

Finally, we note that other transient processes which gave rise to interfering effects were observed in our experiments. The foremost among these was due to the presence of unreacted molecular iodine. Iodine absorbs strongly in the wavelength region where IF gain was probed. This results in an attenuated steady-state probe laser output intensity (recall the discussion from section II). In the wavelength region around $5000 \AA$, where IF was excited, $I_{2}$ absorbs into a continuum and dissociates into atomic iodine, $I\left(5^{2} P_{3 / 2}\right)$, and $I^{*}(5$ $\left.{ }^{2} \mathrm{P}_{1 / 2}\right)^{23,24}$. This was substantiated by the observation of the long lived I* fluorescence at $1.315 \mu \mathrm{m}$ subsequent to the pump pulses. This dissociation reduces the amount of $I_{2}$ in the reactor cell and 
consequently the absorption loss seen by the probe laser is reduced, leading to a transient increase in the probe laser output. In the presence of IF gain these transients appear as long "tails" associated with the true spectrally unresolved IF gain pulses.

Figure 7 shows examples of "gain tails" associated with "thermalized" 0 ' -4 " gain pulses as detected with DS-4. The baseline in these traces represents the steady-state $\mathrm{CW}$ output of the probe laser. Note that as the flow rate of fluorine is increased the magnitude of the tail decreases presumably because less free iodine is present in the flow cell. Note also, however, that even in the lower trace the pulse is still considerably longer than normal for this detection system. The probe laser was set at $6029 \AA$ in these experiments, $4 \AA$ to the blue of the $0^{\prime}-4^{\prime \prime}$ bandhead. The tails are especially pronounced in this example because of the presence of overlapping $\mathrm{I}_{2} \mathrm{~B} \leftarrow \mathrm{X}$ absorption bands at $6033 \AA$ $\left(9^{\prime}-1^{\prime \prime}\right)$ and $6031 \AA\left(11^{\prime}-2^{\prime \prime}\right.$ and $\left.13^{\prime}-3^{\prime \prime}\right)$.

Spectrally resolved gain studies reveal more detail concerning the nature of the "tails". The baseline in such studies represent zero laser intensity. Thus, the presence of the "tail" illustrates that the laser continues to oscillate in the wavelength region to which it was "pulled"

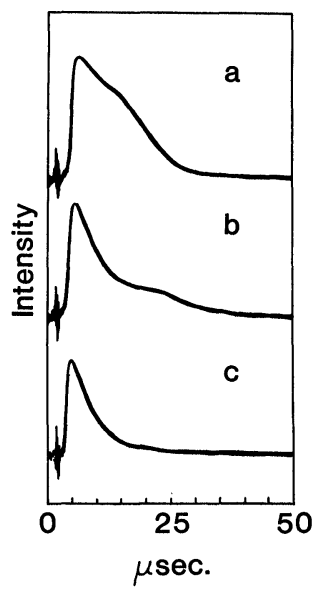

Figure 7 Net (spectrally unresolved) "Thermalized" 0 ' -4 " gain pulse detected with DS-4 (see Figure 4) showing "gain tails" as a function of $F_{2}$ flow rate. (a) $60 \mathrm{sccm} F_{2}$. (b) $150 \mathrm{sccm} \mathrm{F}$. (c) $300 \mathrm{sccm} \mathrm{F} F_{2} . \lambda_{\text {probe }}$ was set $4 \AA$ to the blue side of the $0^{\prime}-4^{\prime \prime}$ bandhead for this experiment, at $6029 \AA$. The baseline in these traces represents the steady-state output intensity of the probe laser. 
for long after the IF gain had decayed. The "tail" can be shortened or lengthened by tuning the probe laser further from or closer to the wavelength at which the gain is monitored. Thus, as one might expect, the losses introduced by the tuning element also play a role in determining the length of gain "tails". Clearly, the period of oscillation of the probe laser at the "pulled" wavelength will shorten as the losses introduced by the tuning element in this region are increased. In our apparatus gain "tails" were rarely observed when the probe laser was set at wavelengths greater than $25 \AA$ from the wavelength at which gain was being monitored, even at relatively high pump powers. An example of a spectrally resolved, "thermalized" gain pulse with a "tail" is shown in Figure 8.

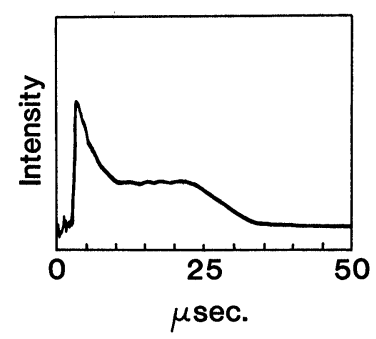

Figure 8 Spectrally resolved "thermalized" gain pulse with "tail." The monochromator of DS-1 (see Figure 4) was set at the $6032 \AA$ corresponding to the 0 ' -4 " bandhead. The probe laser was set at $6022 \mathrm{~A}$. Total cell pressure was 37 Torr. The baseline in this trace represents zero net intensity.

\section{DISCUSSION}

The observation and measurement of small signal gain in IF clearly demonstrates the versatility and relevance of the intracavity gain diagnostic to studies of pulsed gain systems. Perhaps the most interesting facet of our results was the detection of gain from collisionally populated $\left(v^{\prime}, J^{\prime}\right)$ levels in IF. Previously we had observed lasing from $\operatorname{IF}\left(\mathrm{B} ; v^{\prime}=0\right)$ subsequent to having pumped $v^{\prime}$ as large as $6^{16}$. Rapid thermalization via collisions with $\mathrm{He}$ was the mechanism responsible for the vibrational relaxation. In these previous studies there was a delay from the time of excitation to the onset of lasing from $v^{\prime}=0$ which diminished as the He pressure was increased. This delay 
was interpreted as being due to the time required for thermalization and one expects shorter times as the number density of the collision partner $(\mathrm{He})$ is increased. The present results clearly confirm this picture. Since the technique allows one to follow inverted population distributions in time it might be used as a kinetic tool in understanding the energy pathways in excited states of laser systems. However, as illustrated by the results reported in this paper, the usefulness of the technique as a kinetic tool may be limited by the presence of various other mechanisms which give rise to transients in the probe laser output, and care must be taken to characterize these.

The observation that "thermalized" gain is spread across a broad region of the emission band shows that monitoring gain yields much more detailed data than does simply monitoring laser output. In IF when one monitors lasing from $v^{\prime}=0$ at high pressures of $\mathrm{He}$ ( $>5$ Torr) lasing is only seen from $J^{\prime}=21$, the Boltzmann maximum level ${ }^{16}$. Our gain measurements indicated that $v=0$ appeared to be rotationally thermal since gain was seen to monotonically decrease from the bandhead to longer wavelengths. Although we did not pursue this in great detail, it was clear that the population residing in $v^{\prime}=0$, having arrived there via collisions, is spread through many rotational levels. Since lasing was only observed from $J^{\prime}=21$, the level of highest gain, collisional coupling by R-T transfer must couple other $J^{\prime}$ to $J^{\prime}=21$ during lasing. In order to produce efficient lasing in IF via chemical pumping it is essential that $\mathrm{R}-\mathrm{T}$ transfer in the lasing band favorably compete with stimulated emission. Indeed, this $\mathrm{R}-\mathrm{T}$ process may be a limiting kinetic step. The results described here illustrate that one can study this process in a systematic way.

Finally, the approximate gain magnitudes reported here are considerably smaller than the estimated values previously reported for a tranverse cell configuration ${ }^{4,16}$. The "thermalized" gain value reported here for the $0^{\prime}-5^{\prime \prime}$ transition of $\sim 8 \times 10^{-4} / \mathrm{cm}$ is a factor of seven smaller than the previously reported estimate for a transverse flow, while the optimum "direct" gain measured on the 3 '-7" transition $\left(q_{3-7}=0.075\right)^{20}$ was down by more than two orders of magnitude from our previously estimated value reported for the $3^{\prime}-11^{\prime \prime}$ transition $\left(q_{3-11}\right.$ $=0.106)^{20}$. This latter observation cannot be accounted for by the fact that lower pump powers were used in the intracavity gain studies, nor by the inherent inaccuracies in our method of estimating the magnitude of the SSG (see discussion in section II). This is 
substantiated by the failure of our efforts to lase IF on any "direct" transition in our simple longitudinal flow cell configuration, despite the fact that lasing was easily achieved on various "thermalized" transitions in the same flow cell. Because lasing on "direct" transitions is readily obtained in a transverse cell configuration ${ }^{4}$ we were lead to believe that the diminished gain is related to the longitudinal flow configuration. Efforts are currently being made to understand this observation.

\section{CONCLUSIONS}

In this paper we have demonstrated the applicability of the intracavity gain detection technique to the investigation of transient inversions in reactive chemical flows. We have shown that the technique is readily applied to optimization of gain conditions in a flow reactor under conditions not unsimilar to those which may be found in a visible chemical laser system. We have also illustrated that the technique may be applied to gain dynamics and kinetics studies and have pointed out the existence of various transient interference effects which must be dealt with in such applications. The usefulness of the technique is spectrally resolved gain studies of "thermalized" transitions has also been illustrated. Finally, we demonstrated how approximate gain magnitudes may be obtained directly from experimentally obtained data without having to go through the process of making estimates for the various parameters entering into the gain coefficient expression.

\section{References}

1. J. I. Steinfeld, Editor, Electronic Transition Laser I (MIT Press, Cambridge, 1976).

2. L. E. Wilson, S. N. Suchard and J. I. Steinfeld, Editors, Electronic Transition Lasers II (MIT Press, Cambridge, 1977).

3. R. L. Byer, R. L. Herbst, H. Kildal and M. D. Levenson, Appl. Phys. Lett. 20, 413 (1972).

4. S. J. Davis and L. Hanko, Appl. Phys. Lett. 37, 692 (1980).

5. S. B. Hutchison, J. G. Eden and J. T. Verdeyen, Appl. Phy. Lett. 37, 690 (1980).

6. M. Diegelmann, H. P. Grieneisen, K. Hohla, X. Hu, J. Krasinski and K. L. Kompa, Appl. Phys. Lett. 23, 283 (1980).

7. K. A. Truesdell and R. A. Keller, Appl. Opt. 22, 339 (1983).

8. K. A. Truesdell, R. A. Keller and E. F. Zalewski, J. Chem. Phys. 73, 1117 (1980).

9. W. M. Hughes, N. T. Olson and R. Hunter, Appl. Phys. Lett. 28, 81 (1976).

10. J. M. Herbelin, J. A. McKay, M. A. Kwok, R. H. Ueunten, D. J. Spencer and D. J. Benard, Appl. Opt. 19, 144 (1980). 
11. G. C. Bjorklund, Opt. Lett. 5, 15 (1980).

12. D. A. Smith and D. I. Shernoff, Appl. Opt. 24, 1722 (1985).

13. M. B. Klein, Opt. Commun. 5, 114 (1972).

14. M. B. Klein, C. V. Shank and A. Dienes, Opt. Commun. 7, 178 (1973).

15. P. D. Whitefield, R. F. Shea and S. J. Davis, J. Chem. Phys. 78, 6793 (1983).

16. S. J. Davis, L. Hanko and R. F. Shea, J. Chem. Phys. 78, 172 (1983).

17. H. Atmanspacher, H. Scheingraber and C. R. Vidal, Phys. Rev. A 32, 254 (1985).

18. W. Demtröder, Laser Spectroscopy, Springer Series in Chemical Physics, Vol. 5 (Springer, Berlin, 1982).

19. A. L. Bloom, J. Opt. Soc. Am. 64, 447 (1974).

20. M. A. A. Clyne and I. S. McDermid, J. Chem. Soc. Faraday Trans. II 72, 2242 (1976).

21. N. C. Peterson, M. J. Kurylo, W. Braun, A. M. Bass and R. A. Keller, J. Opt. Soc. Am. 61, 746 (1971).

22. T. W. Hänsch, A. L. Schawlow and P. E. Toschek, IEEE J. Quantum Electron. QE-8, 802 (1972).

23. J. Tellinghuisen, J. Chem. Phys. 58, 2821 (1973).

24. S. J. Davis, Appl. Phys. Lett. 32, 656 (1978). 